\title{
An Improvement on C-V Model
}

\author{
Minggang Jing, Jitao $\mathrm{Wu}$, Xiaotao Wang \\ School of Mathematics and Systems Science, LMIB \\ Beijing University of Aeronautics and Astronautics \\ Xueyuan Road 37, Haidian District, Beijing 100191, P.R. China \\ jingminggang130@163.com,wujitao555@buaa.edu.cn,wangxt@126.com
}

\begin{abstract}
C-V model has the advantage of being able to detect boundaries of objects that are not defined by gradient. However, when detecting these types of edges, the $\mathrm{C}-\mathrm{V}$ model only considers the average value of each region without local information. As a result, its segmentation result may exist errors, when detecting non-gradient defined boundaries. In order to overcome this problem, we modify the fitting term of classical $\mathrm{C}-\mathrm{V}$ model with an extra weight. This weight can control the relative height of zero-level contour, so the new method can decrease the segmentation errors. Experiments show that the new model can obtain more accurate results and segment multi-phase images by setting proper weights.
\end{abstract} tation

Keywords-C-V model; Level Set Method; Image Segmen-

\section{INTRODUCTION}

Active contour models [1], based on level set method, are one important type of methods in image segmentation. Those methods can handle topological changes automatically, and can get the boundaries of objects with complex edges. At present, there are two categories of active contour methods. One is based on the edge information and the other is based on the region information. Snakes [2], geometric active contours [3], geodesic active contours (GAC) model [4] are edge-based models. These methods get the boundaries of objects from the gradients of images. As these methods depend on the gradient information, when processing images with non-gradient defined edges, they will get wrong boundaries. While M-S model [5] and C-V model [6] are based on global region information. They take piecewise constant patches as the solution of segmentation problems. As a result, it can process the images whose edges are not well defined. Additionally, C-V model can process images fast and segment the objects with inner-boundaries, and its solution is robust to the initialization of the curves.

However, C-V model may have problems when detecting blurred and fuzzy edges. The reason is that the $\mathrm{C}-\mathrm{V}$ model only considers the averages inside and outside the zero-level contours, and the results determined by its energy functional may be not suitable for these edge types.

In this paper, we propose an improved $\mathrm{C}-\mathrm{V}$ model with a generalized fitting term to deal with the above problem. A weight factor $w$ added into the generalized fitting term, controls the height of zero-level sets of the original $\mathrm{C}-\mathrm{V}$ model. Thus we may obtain accurate segmentation results by choosing an appropriate $w$.

The outline of this paper is as follows. In the next section, We review the classical $\mathrm{C}-\mathrm{V}$ model. In Section III, we present our modification for the $\mathrm{C}-\mathrm{V}$ model. In Section IV, we validate our proposed model by the experimental results and give its analysis. Section $\mathrm{V}$ is the conclusion of this paper.

\section{DESCRIPTION OF C-V MODEL}

In this section, the classical $\mathrm{C}-\mathrm{V}$ model will be reviewed. Let $C$ be a variable curve, inside $(C)$ and outside $(C)$ present the regions inside and outside the curve $C$ respectively. Then the fitting term of the $\mathrm{C}-\mathrm{V}$ model is defined as follows:

$$
\begin{aligned}
F_{1}(C)+F_{2}(C) & =\int_{\text {inside }(C)}\left|u_{0}(x)-c_{1}\right|^{2} d x \\
& +\int_{\text {outside }(C)}\left|u_{0}(x)-c_{1}\right|^{2} d x
\end{aligned}
$$

where $c_{1}$ and $c_{2}$ denote the averages inside and outside the curve $C$ in image $u_{0}$ respectively. Adding the regularizing terms, such as the length of the curve $C$ and(or) the area of the region inside $C$, into the above fitting term, then the classical $\mathrm{C}-\mathrm{V}$ model will be obtained. The energy functional $F\left(c_{1}, c_{2}, C\right)$ is defined by

$$
\begin{aligned}
F\left(c_{1}, c_{2}, C\right) & =\mu \cdot \text { Length }(C)+v \cdot \operatorname{Area}(\operatorname{inside}(C)) \\
& +\lambda_{1} \int_{\text {inside }(C)}\left|u_{0}(x)-c_{1}\right|^{2} d x \\
& +\lambda_{2} \int_{\text {outside }(C)}\left|u_{0}(x)-c_{2}\right|^{2} d x
\end{aligned}
$$

where $\mu, v, \lambda_{1}$ and $\lambda_{2}$ are constants. They can control the influences of their corresponding terms on the energy functional (2). Rewrite (2) into the level set formulation, the $\mathrm{C}-\mathrm{V}$ model will be the following form

$$
\begin{aligned}
F\left(c_{1}, c_{2}, C\right) & =\mu \int_{\Omega} \delta(\phi)|\nabla \phi| d x+v \int_{\Omega} H(\phi) d x \\
& +\lambda_{1} \int_{\Omega}\left|u_{0}(x)-c_{1}\right|^{2} H(\phi) d x \\
& +\lambda_{2} \int_{\Omega}\left|u_{0}(x)-c_{2}\right|^{2}(1-H(\phi)) d x
\end{aligned}
$$

where $H$ is the Heaviside function, and $\delta$ is the Dirac function. As minimizing the above energy functional with respect to $c_{1}, c_{2}$ and , corresponding Euler-Lagrange equations can be written as 


$$
\begin{gathered}
\delta(\phi)\left[\mu \nabla\left(\frac{\nabla \phi}{|\nabla \phi|}\right)-v-\lambda_{1}\left(u_{0}-c_{1}\right)^{2}\right. \\
\left.+\lambda_{2}\left(u_{0}-c_{2}\right)^{2}\right]=0, \\
c_{1}=\frac{\int_{\Omega} u_{0}(x) H(\phi) d x}{\int_{\Omega} H(\phi) d x}, \\
c_{2}=\frac{\int_{\Omega} u_{0}(x)(1-H(\phi)) d x}{\int_{\Omega}(1-H(\phi)) d x} .
\end{gathered}
$$

In order to compute (4)-(5) for the unknown function, we use slightly regularized versions of the function $H$ and $\delta$, denoted by $H_{\varepsilon}$ and $\delta_{\varepsilon}$. After that, it is necessary to discretize the equations with differences implicit scheme. Please refer to [6] for more details.

\section{Our New Proposed Method}

We can conclude that the segmentation of $\mathrm{C}-\mathrm{V}$ model is based on the average intensities in global regions. As we discussed above, the segmentation through $\mathrm{C}-\mathrm{V}$ model may be inaccurate in images whose edges of objects are poorly defined. We will improve the model from its intrinsic property.

\section{A. The improvement on $C$ - $V$ model}

Let I be the image matrix. According to the C-V model, the fitting term (1) is essential for minimizing the energy functional. Suppose that $c_{1}$ and $c_{2}$ are the average values of inside $(C)$ and outside $(C)$ respectively, and $c_{1}>c_{2}$. Then we know that all the pixels whose values are smaller than $\left(c_{1}+c_{2}\right) / 2$ will be classified into the outside $(C)$, on the other hand, the other pixels will locate inside the curve $\mathrm{C}$. That is to say, the optimal solution of the fitting term is controlled by the averages of each region in images. Fig. 1 shows the effect of the fitting term in the C-V model. The fitting term just use the information of averages in different regions. The positions of edges are located about the pixels with values equal to $\left(c_{1}+c_{2}\right) / 2$. As we all know, the real or ideal boundaries of objects may not locate at those pixels most likely.

From the term $-\lambda_{1}\left(I-c_{1}\right)^{2}+\lambda_{2}\left(I-c_{2}\right)^{2}$ in (4), we could get the following equations. And here, we denote it by $T$ for convenience.

$$
\begin{aligned}
T & =-\lambda_{1}\left(I-c_{1}\right)^{2}+\lambda_{2}\left(I-c_{2}\right)^{2} \\
& =-\lambda_{1}\left(I^{2}-2 I c_{1}+c_{1}^{2}\right)+\lambda_{2}\left(I^{2}-2 I c_{2}+c_{2}^{2}\right) \\
& =I^{2}\left(\lambda_{2}-\lambda_{1}\right)+2 I \lambda_{1} c_{1}-c_{1}^{2} \lambda_{1}-2 \lambda_{2} I c_{2}+c_{2}^{2} \lambda_{2} \\
& =\left(I^{2}-c_{1} c_{2}\right)\left(\lambda_{2}-\lambda_{1}\right)+2\left(\lambda_{1} c_{1}-\lambda_{2} c_{2}\right)\left(I-\frac{c_{1}+c_{2}}{2}\right)
\end{aligned}
$$

In the last term of (7), there is a $\left(c_{1}+c_{2}\right) / 2$, we could consider the constant $1 / 2$ as a weight. The general equation form can be rewritten as follows

$$
\begin{gathered}
T_{w}=\left(I^{2}-c_{1} c_{2}\right)\left(\lambda_{2}-\lambda_{1}\right)+ \\
2\left(\lambda_{1} c_{1}-\lambda_{2} c_{2}\right)\left(I-w c_{1}-(1-w) c_{2}\right) \\
=\left(I^{2}-c_{1} c_{2}\right)\left(\lambda_{2}-\lambda_{1}\right)+2 \lambda_{1} I c_{1}-2 \lambda_{1} w c_{1}^{2}-2 \lambda_{2} I c_{2} \\
+2 \lambda_{2}(1-w) c_{2}^{2}+2\left(\lambda_{2} w+\lambda_{1} w-\lambda_{1}\right) c_{1} c_{2} \\
=-\lambda_{1}\left(I-c_{1}\right)^{2}+\lambda_{2}\left(I-c_{2}\right)^{2}+\lambda_{1}(1-2 w) c_{1}^{2} \\
+\lambda_{2}(1-2 w) c_{2}^{2}+(2 w-1)\left(\lambda_{1}+\lambda_{2}\right) c_{1} c_{2} \\
=-\lambda_{1}\left(I-c_{1}\right)^{2}+\lambda_{2}\left(I-c_{2}\right)^{2}+ \\
(1-2 w)\left(\lambda_{1} c_{1}-\lambda_{2} c_{2}\right)\left(c_{1}-c_{2}\right)
\end{gathered}
$$

where $T_{w}$ means the modified term $\mathrm{T}$ with weight $\mathrm{w}$. After we have changed the fitting term, and added $T_{w}$ into (4) instead of $\mathrm{T}$. Then the $\mathrm{C}-\mathrm{V}$ model could be written as

$$
\begin{aligned}
\frac{\partial \phi}{\partial t}= & \delta_{\varepsilon}(\phi)\left[\mu \cdot \operatorname{div}\left(\frac{\nabla \phi}{|\nabla \phi|}\right)-v\right. \\
& -\lambda_{1}\left(u_{0}-c_{1}\right)^{2}+\lambda_{2}\left(u_{0}-c_{2}\right)^{2} \\
& \left.+(1-2 w)\left(\lambda_{1} c_{1}-\lambda_{2} c_{2}\right)\left(c_{1}-c_{2}\right)\right]
\end{aligned}
$$

\section{B. Discussion about how to choose a proper weight}

First, we should fix the coefficient $\lambda_{1}$ and $\lambda_{2}$, and $\lambda_{1}=\lambda_{2}$. Then we could see from (8) that the original fitting term can be written as $2 \lambda_{1}\left(c_{1}-c_{2}\right)\left(I-w c_{1}-(1-w) c_{2}\right)$. Without loss of generality, we set $\lambda_{1}=\lambda_{2}=1$, so we get $T_{w}=T+(1-2 w)\left(c_{1}\right.$ $\left.-c_{2}\right)^{2}$. The above weight $w$ controls the influence of the inner or the outer areas of the curves on the final segmentation result. The weight should be in the interval $[0,1]$. The smaller $w$ indicates the less affection of the corresponding region inside $(C)$ in the model.

Obviously, when $w$ is equal to $1 / 2$, our model is just the classical C-V model. From the gradient descending flow (10), we could conclude that if $w$ is close to 0 (or $0<=w<1 / 2$ ), the value of the solution will be larger than that of the classical C-V model. The reason is $(1-2 w)\left(c_{1}-c_{2}\right)^{2}>0$, and $\partial / \partial t$ is greater than that of original $\mathrm{C}-\mathrm{V}$ model. So the region of $>0$ will be larger and the region of $<0$ will be smaller. We have supposed $c_{1}>c_{2}$, so the smaller $\mathrm{w}$ is, the smaller $c_{1}$ will be.

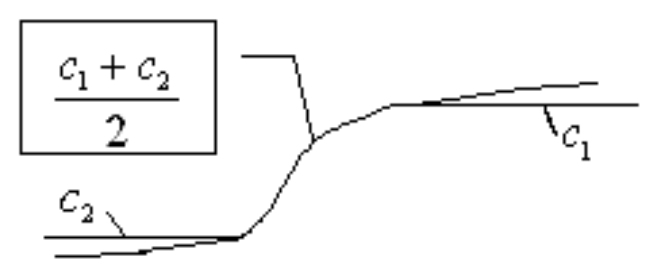

Figure 1. Projection of image 


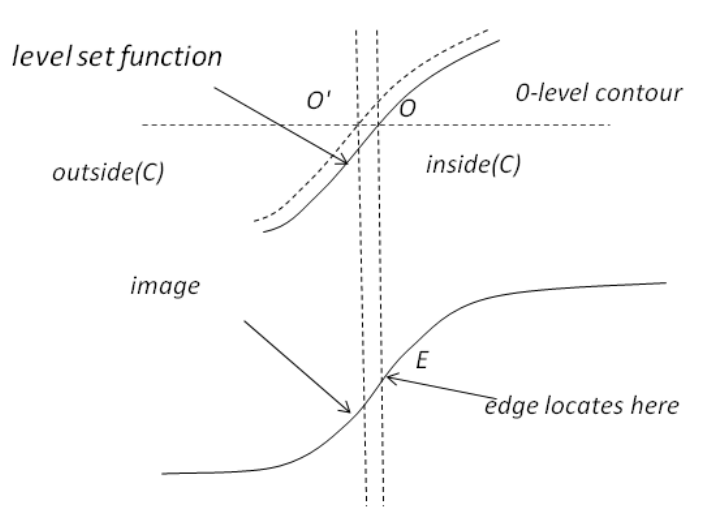

Figure 2. Explanation of diminishing the weight w. With the weight descending, the final solution is increasing. So the zero point $O$ (the intersection the zero-level and ) is moving left and the area of inside $(C)$ is growing.

That is to say, the large $w$ has greater effect on the region inside $(C)$. That is because the region of inside $(C)$ will be larger, more pixels with value smaller than $c_{1}$ will be added into the region, and the average goes down too. On the contrast, the larger $w$ is, the smaller region area of $>0$.

Fig. 2 shows the relationship between the level set function and the segmented regions (or edges). If is growing, i.e. the weight $w<1 / 2$ is chosen, the intersection point $O$ will move towards $O^{\prime}$ and the edge, which is defined by the zero-level contour, will move left too. So we could see clearly how the weight $w$ influences the segmentation result.

It is necessary to discuss how to choose the weight $w$. All the discussions suppose that the contour function is larger than zero inside the zero-level contour. So when we use the small weight $(0<=w<1 / 2)$, the area of the inner region will be larger than the classical C-V model. But another factor we should consider is the luminance of the object. Whether it is larger or less than that of the background. If the real boundary is larger (smaller) than the value $\left(c_{1}+c_{2}\right) / 2$ and the intensity of the object is larger (smaller) than the background, we should choose large weight $w$. Otherwise, we should choose small weight $w$. In other words, the large weight $w$ will guide the 0 -level contour moving towards up.

\section{EXPERIMENTAL RESULTS AND ANALYSIS}

In this section, we will show some experimental results that are obtained from our proposed method. The first example is a medical image, as the medical images have fuzzy edges, it will be more clearly to watch the differences between our method and the classical $\mathrm{C}-\mathrm{V}$ formulation. The second experiment is tested on an synthetic image, which has four objects with different constant intensities.

Although, the classical $\mathrm{C}-\mathrm{V}$ model has the ability to capture the interesting areas by those two parameters $\lambda_{1}$ and $\lambda_{2}$. But those two parameters' range is the right half of the real line, and it is difficult to decide or choose the proper parameters. The solution is sensitive to $\lambda_{1}$ and $\lambda_{2}$. Sometimes, when we change $\lambda_{1}$ or $\lambda_{2}$, we have to change other parameters (e.g. $\mu$ ) in C-V model to get a solution. So they could be properly chosen possibly, but it is a difficult work.

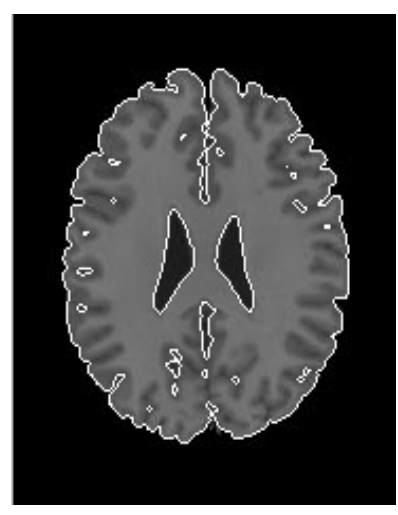

(a) The result of the proposed method with $w=0.64$

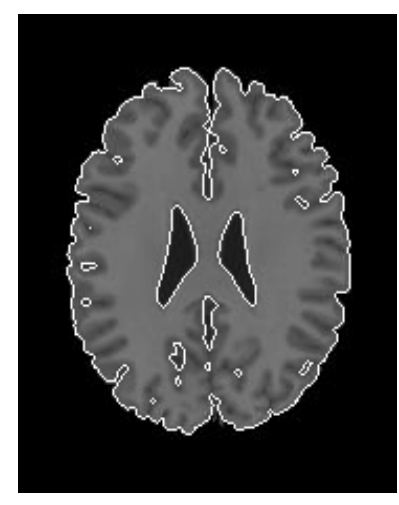

(c) The result of C-V model with $\lambda_{1}=0.2, \lambda_{2}=1$

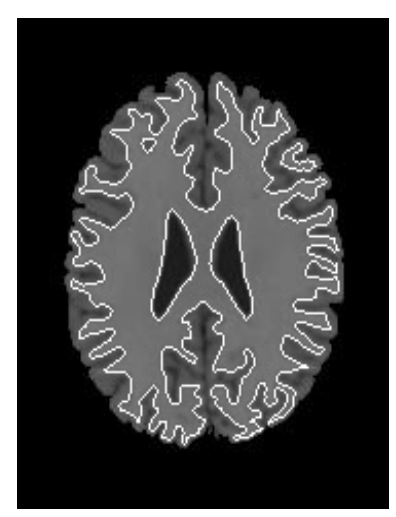

(b) The result of the proposed method with $w=0.83$

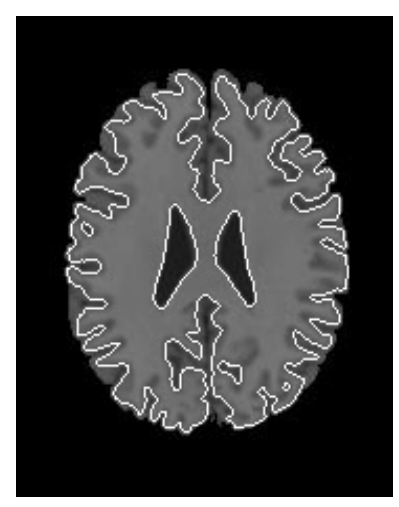

(d) The result of $\mathrm{C}-\mathrm{V}$ model with $\lambda_{1}=1, \lambda_{2}=0.32$
Figure 3. The results obtainted from our method and the classical C-V model on an MR image. The white curves are the edges detected by corresponding methods.

Fig. 3 shows the experimental results of our proposed method and C-V model on an MR image of brain. In Fig. 3 (a) and (b) show that our proposed method can detect different objects with different weight $w$. (a) shows the segmentation result of the whole brain with $w=0.64$, while (b) shows the detection of cerebral white matter $w=0.83$. And these two results obtained by $\mathrm{C}-\mathrm{V}$ model are showed in (c) and (d), and the pairs of $\left(\lambda_{1}, \lambda_{2}\right)$ are $(0.2,1)$ and $(1,0.32)$ respectively. With $\lambda_{1}>\lambda_{2}, \mathrm{C}-\mathrm{V}$ model has a greater tolerance for the differences of image intensities inside $\mathrm{C}$ than outside C. It is contrary for $\lambda_{1}<\lambda_{2}$. (a) and (c) show that those two results are similar to each other, but edge gotten from $\mathrm{C}-\mathrm{V}$ model is smoother especially at the top center. And several chinks between grey matter are not segmented from the brain region. That is to say some of areas in the background are segmented into the object area obtained by $\mathrm{C}-\mathrm{V}$ model. Obviously, there are more differences between (b) and (d). And the result (b) is more accurate than result (d).

Fig. 4 shows the multi-phase segmentation results obtained through the proposed method on a synthetic image. 
There are four objects in this image - two squares with the intensities 80 and 150, and two round plates with the intensities 75 and 155 respectively. And the luminance of background is 60 . In this experiment, there is a great topological change, that is the inner

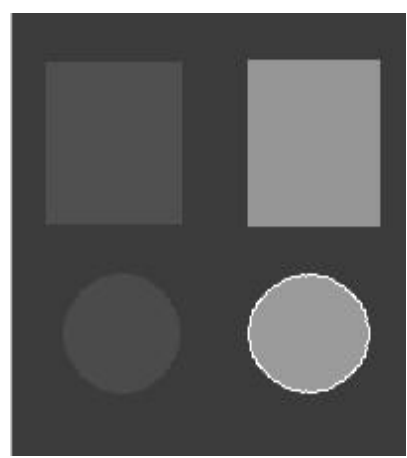

(a) The result of the proposed method with $w=0.01$

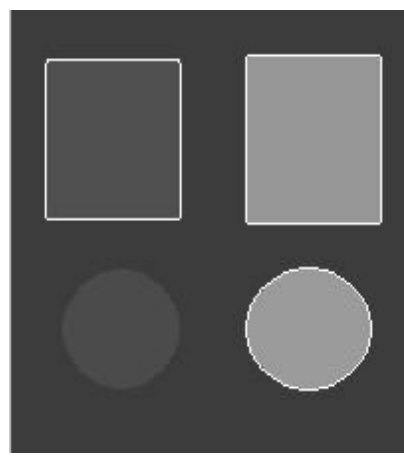

(c) The result of the proposed method with $w=0.73$ Figure 4. The results obtainted from the method in this paper. The white
curves are the edges.

and outer region of zero-level contour changing their signs in the iterative procedure. So the selection of the weight should be contrary to what we have discussed in the previous section. (a) shows the segmentation of the object with the highest intensity among these four objects. If we change the weight $w$ in the proposed method, we could also get two, three, and four objects in this image, as shown in Fig.4(b)-(d).
But there is a difficulty for $\mathrm{C}-\mathrm{V}$ model to segment one or three objects. The reason is that, there are two pairs of objects with very similar intensities. It is difficult for $\mathrm{C}-\mathrm{V}$ model to capture this slight difference. The proposed method can detect the objects in this image accurately.

\section{CONCLUSION}

The comparison between algorithms, that are based on gradient information, and $\mathrm{C}-\mathrm{V}$ model shows that $\mathrm{C}-\mathrm{V}$ model has the advantage of detecting object with non-gradient defined edges. But $\mathrm{C}-\mathrm{V}$ model just takes account of the global zero-level contour. As a result, the segmentation results may be unideal or inaccurate. In this paper, we have analyzed the $\mathrm{C}-\mathrm{V}$ model and improved it with adding a weight into the classical model. The discussion of how to choose the proper weight is also presented in this article. So it may need the supervision to choose a better weight.

Two experiments are presented in this paper. The first experiment shows our method can detect objects with blurred or fuzzy edges. The second experiment shows the proposed method can segment multi-phase images, that contains objects with very similar intensities. But $\mathrm{C}-\mathrm{V}$ model has difficulties to segment objects in those experiments. And those experimental results show that the proposed method outperforms $\mathrm{C}-\mathrm{V}$ model.

\section{ACKNOWLEDGMENT}

This work is supported by Key Projects in National Science in the National Science and Technology Pillar Program ( 2008BAC34B03-4)

\section{REFERENCES}

[1] S. Osher and J. A. Sethian, "Fronts propagating with curvature dependent speed: Algorithms based on Hamilton-Jacobi Formulation," J. Comput. Phys., vol. 79, pp. 12-49, 1988.

[2] M. Kass, A. Witkin, and D. Terzopoulos, "Snakes: Active contour models," Int. J. Comput. Vis., vol. 1, pp. 321-331, 1988.

[3] V. Caselles, T. Catté, T. Coll, and F. Dibos, "A geometric model for active contours in image processing," Numer. Math., vol. 66, pp. 1-31, 1993

[4] V. Caselles, R. Kimmel, and G. Sapiro, "On geodesic active contours,” Int. J. Comput. Vis., vol. 22, no. 1, pp. 61-79, 1997.

[5] D. Mumford and J. Shah, "Optimal approximation by piecewise smooth functions and associated variational problems," Commun. Pure Appl. Math, vol. 42, pp. 577-685, 1989.

[6] T. F. Chan and L. Vese, "Active contours without edges," IEEE Transactions on Image Processing, vol 10, no. 2, pp. 266-277, 2001 